\title{
NORMALITY AND THE NUMERICAL RANGE OF AN OPERATOR ${ }^{1}$
}

\author{
BY J. G. STAMPFLI
}

Communicated by L. Nirenberg, June 28, 1966

For an operator $T$ on a Hilbert space $H$, let $W(T)=\{(T x, x): x \in H$ and $\|x\|=1\}$ denote the numerical range of $T$; and let $\Sigma(T)$ designate the convex hull of $\sigma(T)$, the spectrum of $T$.

It is well known, for a normal operator $T$, that $\operatorname{cl} W(T)=\Sigma(T)$. Our main results are, in a certain sense, converses to this statement. They generalize results of Donoghue [1], Hildebrandt [2], Nieminen [4], Meng [3], Putnam [6], and the author [7].

I. First, some definitions. A curve $C$ in the complex plane is convex if it has a support line at every point. If, at every point, the curve and the origin lie on the same side of the support line, the curve is said to be convex with respect to the origin.

A curve $C \in \mathcal{e}$, if

(1) $C$ is given by a single valued function $R=f(\theta)$, where $R>0$ for $\delta_{1} \leqq \theta \leqq \delta_{2}$,

(2) both $C$ and $C^{-1}(R=1 / f(-\theta))$ are convex,

(3) both $C$ and $C^{-1}$ are convex with respect to the origin.

Membership in the class $\mathcal{C}$ is not intrinsic to a curve. For example, it is not preserved under translation. Roughly speaking however, the curves in $\mathfrak{e}$ may be said to be "flat" convex curves.

The first of our main results is

Theorem 1. Let $\sigma(T)$ lie on a curve $C \in \mathrm{e}$. Then $T$ is normal if and only if $W\left(T^{ \pm 1}\right) \subset \Sigma\left(T^{ \pm 1}\right)$.

Since the unit circle is clearly in $\mathcal{C}$, as an immediate consequence, we obtain

Theorem A [Donoghue]. If $\left\|T^{-1}\right\| \leqq 1$ and $W(T)$ is contained in the unit disc, then $T$ is unitary.

The following sharper version of Donoghue's theorem is also a corollary of Theorem 1.

Theorem B. If $T$ is invertible and both $W(T)$ and $W\left(T^{-1}\right)$ are contained in the unit disc, then $T$ is unitary.

Theorem 1 may be said to be best possible in the following sense. There exists an operator $T$ where

1 This work was partially supported by the National Science Foundation, under grant number GP-5152. 
(1) $\sigma(T)$ is given as a single valued function $R=f(\theta)$;

(2) both $\sigma(T)$ and $\sigma\left(T^{-1}\right)$ are convex curves;

(3) both $\sigma(T)$ and the reflection of $\sigma\left(T^{-1}\right)$ with respect to a support line, are convex with respect to the origin;

(4) $W\left(T^{ \pm 1}\right) \subset \Sigma\left(T^{ \pm 1}\right)$.

However $T$ is not normal.

Despite this, the following modification of Theorem 1 is valid.

Theorem 2. Let $\sigma(T)$ lie on a smooth convex curve. If

$$
W(T) \subset \Sigma(T)
$$

and

$$
W\left[(T-z I)^{-1}\right] \subset \sum\left[(T-z I)^{-1}\right], \quad \text { for } z \notin \sigma(T),
$$

then $T$ is normal.

Here, smooth means a continuous second derivative. Note that even though $S$ is a convex curve, in general $S^{-1}$ is not. Thus $\Sigma\left[(T-z I)^{-1}\right]$ may engulf $\sigma\left[(T-z I)^{-1}\right]$ at many points.

From this theorem, we may deduce

Theorem C [Putnam, Stampfli]. If $T$ is hyponormal, and $\sigma(T)$ lies on a smooth convex curve, then $T$ is normal.

Because of the many conditions, short of normality, which are known to imply $W(T) \subset \Sigma(T)$ (see [2], [5], and [9]), there are numerous other corollaries of Theorems 1 and 2. These, and the proofs, will appear elsewhere [8].

\section{REFERENCES}

1. W. F. Donoghue, On a problem of Nieminen, Inst. Hautes Études Sci. Publ. Math. 16 (1963), 127-129.

2. S. Hildebrandt, Über den numerischen Wertebereich eines Operators, Math. Ann. 163 (1966), 230-247.

3. C. H. Meng, On the numerical range of an operator, Proc. Amer. Math. Soc. 14 (1963), 167-171.

4. T. Nieminen, $A$ condition for the self-adjointness of a linear operator, Ann. Acad. Sci. Fenn. Ser. A. I No. 316 (1962), 3-5.

5. G. Orland, On a class of operators, Proc. Amer. Math. Soc. 15 (1964), 75-80.

6. C. R. Putnam, On the spectra of semi-normal operators, Trans. Amer. Math. Soc. 119 (1965), 509-523.

7. J. G. Stampfli, Hyponormal operators and spectral density, Trans. Amer. Math. Soc. 117 (1965), 469-476.

8. - Minimal range theorems for operators with thin spectra, Trans. Amer. Math. Soc. (to appear).

9. J. Williams, Spectral sets and finite dimensional operators, Ph.D. Thesis, Univ. of Michigan, Ann Arbor, Michigan, 1965.

New York UnIVERSITY 